\title{
Intelligent Transportation System for Developing Countries - A Survey
}

\author{
Gurdit Singh \\ PhD Scholar \\ PEC University of Technology \\ Chandigarh
}

\author{
Divya Bansal, Ph.D \\ Associate Professor \\ PEC University of Technology \\ Chandigarh
}

\author{
Sanjeev Sofat \\ Professor \\ PEC University of Technology \\ Chandigarh
}

\begin{abstract}
India is the second largest country in the category of population. With the increasing population the consumption of the vehicles also increases, leading to critical burden on traffic management in the metropolitan cities and towns of the country. In 2011-12 alone, 20.4 million motorized vehicles were sold in India leading to congestion and accidents on the roads. For controlling the traffic on the road some traditional methods were used like using traffic lights and traffic signs, traffic policemen and round-about. In this paper we will discuss the existing techniques which will help India for controlling the traffic on the roads.
\end{abstract}

\section{General Terms}

ITS, Sensors, ITS Technology.

\section{Keywords}

ITS, Sensors, ITS Techniques, ITS Technology, ITS Application.

\section{INTRODUCTION}

Traffic is the major concern in the developed and developing countries. Every country have their rules and regulations to control the traffic. In the past decade the density of vehicles had increased on the roads leading to road traffic, congestion and accidents. Traditional methods were used for controlling the traffic i.e. deploying traffic lights, traffic signs, traffic policemen and round-about. But these methods are getting obsolete day by day. In the era of technology, intelligent and adaptive equipment should be used to control the traffic.

In majority, traffic is controlled by the Intelligent Transportation System (ITS) [1]. Intelligent Transportation System is a computerized system having diverse applications connected with Vehicle Transportation, which gets the live feeds from the sophisticated sensors of the smartphone and the equipment installed on the road side or with camera mounted on the traffic light poles. Smartphones consists of high end processor and many sophisticated sensors in built. Sensors like GPS, Accelerometer, Proximity, Gyro meter, Microphone and Camera makes the smartphones different from other phones. ITS is being used and deployed in developed countries for real time navigation, traffic updates, lane discipline and predicting travel time. Its goal is to improve effectiveness, efficiency and safety of the road transportation system. ITS had been successfully deployed in various developed countries like Japan, South Korea, Singapore, United States, Australia and United Kingdom. Deployment of ITS is different in different countries, but the motive is same i.e. to improve the transportation system performance including reduced congestion, increased safety and traveler convenience.
The transportation model differs from country to country. India comes under the category of the developing countries and hence the transportation scenario is different from the developed countries. ITS assists the vehicles to move in a particular lane in developed countries i.e. vehicles should follow the lane disciplines but in contrast with India, the single road is shared by different types of vehicles i.e. 2wheelers, 3-wheelers or 4 wheelers motorized or nonmotorized, thereby increasing the congestion on the road. Some preventive measures to control the traffic were taken like by placing the cameras [21] on the traffic light poles and RF-sensors along the road side or being guided by traffic policemen. But these were all controlled manually and thus not assisting the vehicle in real time. The solution to this approach is to use transportation model specially developed for developing countries.

ITS development has been driven strongly by socio-economic needs and environmental demands. There are some lacking points which make the deployment of ITS hard in India i.e. geography and the economic growth, population density and cultural factors. In India, the diverse range of vehicular velocities (pedestrian, bicycle, LMV's, HMV's, animal carts), wide variety of vehicles (including pedestrian traffic) and poor lane discipline (partially resulting from the first two factors and partially due to cultural reasons) and a very high population density makes adoption of Western ITS standards and architecture difficult to deploy. The Indian ITS must be designed to suit the Indian scenario by modifying the ITS protocol through some research.

In the later section of the paper, existing research work is discussed on the basis of case studies of mobile computing in transportation field. Section III discusses about the benefits of using ITS, later section IV discusses about the applications of ITS. Use cases of worldwide leaders with their technologies used in the implementation of ITS are being presented in section V. In section VI the various communication techniques are presented. In section VII the need of ITS in India and solution approach for reducing problems of transportation are suggested for India.

\section{RELATED WORK}

Congestion on the road is the major issue now these days. In India there are several reasons which attribute to high level of congestions [6] on the roads. The single road is shared by different types of vehicles i.e. 2-wheelers, 3-wheelers or 4 wheelers motorized or non-motorized vehicles. There by creating a congestion. In developed countries the specific lane is meant for specific transport vehicle following lane discipline. So there is less chance of congestion on the roads. In the developed countries like in Japan, South Korea, Singapore, United States, Australia and United Kingdom ITS [14] had played an important role, and aided in reduction of 
congestion on the roads and improve traveler's experience. While moving on the road if there is a congestion with say accident, then commuters need be informed that there is congestion ahead.

Some research work has already been done by the different authors to reduce the congestion and to control the traffic. According to the authors there are several reasons which can create the congestion on the roads. For the detection of the congestion the various authors suggested to use smartphones, which has various sophisticated sensors in built. Through the smartphone's sensors we can collect the information about the particular place.

Potholes are the major concern and can be seen on any road. It grows in size day by day if it is not properly maintained. $\mathrm{P}$. Mohan, Eriksson et.al. [3, 4] suggested to get the position of pothole and send the information to the central server, sensors like Accelerometer, GPS and GPRS are used of the smartphone. When the vehicle goes into the pothole, variation in the accelerometer is noted and GPS value will be recorded on that particular location. The whole information is sent to the central server via GPRS where the data is analyzed and stored in the database. When some another vehicle is moving on that same road, the vehicle will be notified thorough an application that there is a pothole ahead. If the pothole exists for the longer time which creates congestion on the roads, will be notified to the construction company for repairing of the road.

Every person honks other person's vehicle to get the side or space on the road. Too much honking on the road means there is congestion ahead. R. Sen, S. Roy et al. [1,5] suggested to find the level of congestion on the road. Two microphones are implemented on the road side to detect the honks. The microphones are placed at some distance apart. The frequencies between the two honks is noted and their difference will tell the level of congestion on the roads. If the difference between the two frequencies is too small or same then it is notified that there is a congestion on the road.

An experiment had been done inside the IIT Campus. R. Bhoraskar et al [6] calculated the density of congestion inside IIT Campus. An application is designed in such a way that it gets the input from the various sensors present in the smartphone to sense honks, speed-breakers, and the breaking events. After collecting all the data from the smartphone, data will be sent to central server which can use the information received to annotate maps accessed by the other users through an application. This application can contain lot of information like the intensity of traffic at an intersection, the bumpiness on the roads etc.

The road chaotic conditions is due to congestion which can be happened at any time. The passengers on the bus stop has to wait for long time. J.Biagioni et al. [2] solution predicted the location and arrival time of the bus. The experiment is done by setting an application on bus so that tracking of the bus is easy. This application will also locate another bus nearby and send the information to the central server to track down another bus also. This makes the server to keep record of each and every bus on the track and predicted arrival time. At the bus stop the user can get all the information about the various bus arrivals at the spot.

Sensor like GPS also plays an important role for finding the exact location of the device. But it is battery hungry. The regular use of the GPS over a long time may drain the battery at the faster rate. A.Thiagarajan et al. [8] suggested that GPS signals are weak and will not be able to work when it inside pocket, inside house or inside tunnel. There is an alternative to GPS is to use Wi-Fi. Using the Wi-Fi there are some benefits like the battery will not drain quickly as GPS and another reason is, it will give us the approximate location up to 40 meters of radius.

Using GPS we can find out the location but we cannot find the history of the place or navigate the vehicle on the road with real time traffic. P. Angin et al. [7] suggested to use camera sensor present in the smartphone to get navigated and assisted in the real time. The camera will give the live feeds to the central server, where in response the server will analyze and predict the traffic on the road. The scenario is best fitted for the blind persons while crossing the road. The camera will be mounted on the person and gives the live feeds to the server. In return the server will assist the blind person when to cross the road or move along the road when there is no traffic. The processing is done on the image of video to extract the traffic light color and warns the blind person. The data should be sent and processed in a real time so that blind person may get the live feeds. Sending the data to the central server requires network to be maintained.

In the 21 st century, mostly user rely on the smartphones where they get updated all the time. Live feeds will be sent to the user for which he is subscribed to. J. Chen et al. [9] suggested for getting the live feeds from the central server the user must the first subscribed to the service. Like using the services of Facebook or Twitter, the user should be subscribed to the services first, after subscription he can easily publish or subscribe the post. The user can obtain information without regard of their location or source.

All of the publishing and subscription is maintained by the central server which maintains a record of each and every user to which he is subscribed to. Going internally V. Jacobson et al. [17] suggested for publishing and subscribing services the model NDN is concerned. The model NDN (Named Data Networking) is based on Content-Centric Network (CCN) [17] where the data is stored centrally. Regarding the subscription the whole structure is divided into four parts i.e. first is Content store, where the data is stored centrally at the server. Second is Pending Interest Table (PIT), where the requests had been queued up. Third is Forwarding Tables (FIB), where the updates of the content are stored per user and fourth is the Subscription Table (ST) which stores all the subscriptions of the user. The user will get the feeds corresponding to the subscribed data.

The feeds should be sent so fast such that there should be negligible delay. The data flow to each and every hardware that is router or the switches, communicate using XML language. W. Fenner, M.Ott et.al. [10, 11] suggested that, to query for accessing the data from the database of the subscription should be done using XML. The user will send a query through an API, which in turn convert the user's query in XML format and send to the central server and in response to the query a reply will be sent to the user in XML format. The data passing from one hardware to another is also done using XML language.

The central server will be overloaded when more and more queries from the user arrives. With this the response time be slow and thus the user has to wait. T. Hunter et al. [12] suggested that instead to use single server, the whole architecture should be shifted to cloud infrastructure, where multiple servers share their resources and speeding up the 
computation. Thus thereby decreasing the time of computation and resources.

The traffic on the road changes dramatically every minute thereby increases the incidents. Wang et al [22] suggested a hybrid approach for automatic incident detection. A new approach is defined where time series analysis and machine learning are used. The machine learning component aims to detect real time traffic, predicted normal traffic as well as the difference between two. They collected the data from the static sensors, dynamic sensors and traffic cameras.

Bret Hull et al. [16], proposed a computing system which is designed to collect, process and deliver and visualize data from the sensors located in smartphones. The data from the sensors sent to the central server wirelessly (Wi-Fi, Bluetooth) where the data is stored in the database for the further analysis and visualizations. There are nodes situated at the road side and will carry the data coming from the mobile nodes that is from the mobile phones.

\section{KEY BENEFITS OF USING ITS}

Most of the developed country's transportation had been assisted and directed using ITS. The main motive of the ITS is to provide the public safety, environmental feeds and many more. Some of the other key benefits of using ITS are as follows:

\subsection{Increasing Safety}

The main motive of using ITS is to provide the public safety on the roads. In the past several years the trend of driving had been changed, thereby increasing the road accidents. So to avoid or get guided before any incident to happen the ITS plays a vital role. ITS will guide the user through voice as well as gives an alert to the user about the traffic or congestion ahead, so that the user may act accordingly. With this the ratio of unwanted accidents will be decreased to some extent.

\subsection{Delivering environmental benefits}

The major benefit of ITS is to deliver the environment alerts if any. The sensors are being placed along the road side so that they can record the temperature, humidity and other environmental factors. Calculating all the factors, the values will be sent to the central server where the values are been stored in the database. If any user is subscribed to this activity he may get an alert about the environment. The forecast and precast weather report will also be sent to the user.

\subsection{Capacity / Throughput}

ITS also keeps a track on the congestion before it took place. All it is done by getting the number of vehicles on each and every lane. If the number of vehicles are more on single lane, then ITS takes a decision to route the traffic onto another lane. Thus decreasing the risk of congestion of the particular lane. It also keeps tracks on the capacity of the vehicles running on the road at a particular time on particular lane. It will also send an alert to user that at some particular time the congestion will feel high on some particular lane.

\subsection{Information Dissemination}

Delivering the report of the road about the congestion there are many other ways to present to user such as using Dynamic Message Signs (DMS), Variable Message Signs (VMS) and Highway Advisory Radio (HAR).

\subsection{Enhancing mobility and convenience}

Intelligent transportation system can be enhanced in satellite based vehicle navigation and various other application which can deliver the real time traffic information to the user. User can check the status of the traffic and other information where they are heading before departing from their homes.

\section{APPLICATIONS / SERVICES / FUNCTIONAL AREAS OF ITS}

There are some applications associated with the use of ITS which are as follows:

Table 1. Applications and Categories of ITS

\begin{tabular}{|c|c|c|}
\hline S.No. & ITS Category & $\begin{array}{l}\text { Specific ITS } \\
\text { Applications }\end{array}$ \\
\hline 1. & $\begin{array}{l}\text { Advanced Traveler } \\
\text { Information System } \\
\text { (ATIS) }\end{array}$ & $\begin{array}{l}\text { - Real-time Traffic } \\
\text { Information } \\
\text { - Route Guidance / } \\
\text { Navigation Systems } \\
\text { - Roadside Weather } \\
\text { Information Systems } \\
\end{array}$ \\
\hline 2. & $\begin{array}{l}\text { Advanced } \\
\text { Transportation } \\
\text { Management Systems } \\
\text { (ATMS) }\end{array}$ & $\begin{array}{l}\text { - Traffic Operations } \\
\text { Centers } \\
\text { - Dynamic Traffic Signs }\end{array}$ \\
\hline 3. & $\begin{array}{l}\text { ITS-Enabled } \\
\text { Transportation } \\
\text { Pricing Systems (ITS- } \\
\text { ETPS) }\end{array}$ & $\begin{array}{l}\text { - Electronic Toll } \\
\text { Collection } \\
\text { - Variable Parking Fees }\end{array}$ \\
\hline 4. & $\begin{array}{l}\text { Advanced Public } \\
\text { Transportation } \\
\text { System (APTS) }\end{array}$ & $\begin{array}{l}\text { - Real-time Status } \\
\text { Information for Public } \\
\text { Transit System } \\
\text { - Automatic Vehicle } \\
\text { Location }\end{array}$ \\
\hline 5. & $\begin{array}{l}\text { Fully Integrated } \\
\text { Intelligent } \\
\text { Transportation (FIIT) }\end{array}$ & $\begin{array}{l}\text { - Collision Avoidance } \\
\text { - Intelligent } \quad \text { Speed } \\
\text { Adaptation }\end{array}$ \\
\hline 6. & $\begin{array}{l}\text { Advanced Traffic } \\
\text { Management System } \\
\text { (ATMS) }\end{array}$ & 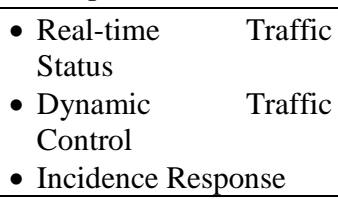 \\
\hline 7. & $\begin{array}{l}\text { Commercial Vehicle } \\
\text { Operations (CVO) }\end{array}$ & $\begin{array}{l}\text { - Traceability and safety } \\
\text { of commercial vehicles } \\
\text { such as trucks, vans, } \\
\text { and taxis. }\end{array}$ \\
\hline 8. & $\begin{array}{l}\text { Advanced Vehicle } \\
\text { Control Systems } \\
(\text { AVCS) }\end{array}$ & $\begin{array}{l}\text { - Collision Warning of } \\
\text { the vehicles }\end{array}$ \\
\hline 9. & $\begin{array}{l}\text { Advanced Rural } \\
\text { Transportation } \\
\text { System (ARTS) }\end{array}$ & $\begin{array}{l}\text { - Provide Information } \\
\text { about Remote roads } \\
\text { via Radio. }\end{array}$ \\
\hline
\end{tabular}

\section{WORLD WIDE LEADERS OF ITS}

ITS had been deployed in various developed countries and some of the major countries which are world-wide leaders in the field of Intelligent Transportation System which are as follows:

\subsection{Japan}

Japan leads the world in Intelligent Transportation System based on the importance of acceptance of ITS in Japan, citizens get benefits of deployed ITS applications and 
maturity of those applications. The goal of ITS is to provide the real time information about the traffic conditions. Real time information can be collected using two techniques. One is to embed fixed sensors in or on the road sides, which was started in 1996 and the second is via mobile probes like taxis or mobile phones, which was started in 2003.

\subsection{South Korea}

The strength of South Korea in the ITS application makes the world leader in Intelligent Transportation System. The strengths include the real time traffic information, advanced public transportation and electronic fare payment. The South Korean's Expressway Management Systems collects the traffic information via Vehicle Detection Systems, which is installed on the roadside at $1 \mathrm{~km}$ of intervals. Second is Closed Circuit Camera deployed every $2-3 \mathrm{~km}$. and last through probe vehicles. The data is collected and stored at South Korean's National Transport Information Center where the data is disseminated to other users via various communication means.

\subsection{Singapore}

Singapore is a world leader in Intelligent Transportation Systems based on the use of probe vehicles to collect the real time traffic information, road pricing, and deployment of computerized traffic signals national wide and the use of ITS applications. Singapore's ITS will deliver the services like location based and traffic information to the commuters through in-vehicle devices or advances congestion management systems which will target the pedestrian and variable user on the roads.

\subsection{United States}

United States Department of Transportation coordinates the ITS through Research and Innovative Technology Administration (RITA) wing. US-ITS specially focus on Telephonic Data Dissemination, IntelliDrive, Next Generation 9-1-1, Cooperative Intersection Collision Avoidance Systems, Congestion Initiative, Integrated Corridor Management Systems, Clarus Initiative, Emergency Transportation Operations, Mobility Services for All Americans and Electronic Freight Management [20].

\subsection{Australia}

Australia [13] also leads in the field of Intelligent Transportation System. The main motive of Australian's ITS is to improve the traffic scenarios and enhancing the public safety. The project team of Australian's ITS recommended to adopt FRAME as its architecture basis so that future enhancements and additions can be easily adopted both in Australia and FRAME.

\subsection{United Kingdom}

United Kingdom also leads world in Intelligent Transportation System [15]. United Kingdom used the MIRA Intelligent Transportation System as its base ITS architecture. MIRA uses the military grade self-healing communications networks technologies and topologies, MIRA is able to ensure safe operation of very complex control systems.

\section{COMMUNICATION TECHNIQUES}

Following are the communication techniques which can be used in ITS by using various sensors of the smartphone. Some of the sensors are listed below:

\subsection{GPS (Global Positioning System)}

It is the satellite based navigation that provides the location and time. 4 or more GPS satellites are used to find the location of the device. Each satellite continually transmits messages that include the time and position when the message was transmitted.

\subsection{Wi-Fi (Wireless Fidelity)}

Wi-Fi is based on IEEE 802.11 standard which is also defines Wireless Local Area Network (WLAN). Till now every smartphone and network devices even personal computers are equipped with WLAN. With the shortage in the range it is basically used in limited space.

\subsection{Microphone}

It is an electric sensor which converts sound into electrical signals. They are basically used in telephones, hearing aid, tape recorders. In the smartphones it plays a vital role in communication.

\subsection{Camera}

Every smartphone is equipped with an inbuilt camera to record or take the images and videos. We can extend the use of camera in two strategies. One is to manually monitor the traffic by mounting a camera on the traffic light poles and other can be used for the blind persons [7], who are visually impaired. In Delhi [21], the real time traffic is manually monitored using cameras at different places where mostly congestion takes place.

\subsection{Accelerometer}

Accelerometer is an electronic device which measures tilt and motion of the component. It is also capable of detecting rotation and motion gestures such as swinging or shaking. Every smartphone has this component inbuilt thereby can check the rotation and motion of the device.

\subsection{Gyroscope}

Gyroscope is a device which is used for measuring and maintaining orientation based on the principal of angular momentum. Mechanically, a gyroscope is a spinning wheel or disc in which the axle is free to assume any orientation. Although this orientation does not remain fixed, it changes in response to an external torque much less and in a different direction than it would without the large angular momentum associated with the disc's high rate of spin and moment of inertia. The device's orientation remains nearly fixed, regardless of the mounting platform's motion, because mounting the device in a gimbal minimizes external torque.

\subsection{Magnetometer}

The magnetometer is a measuring instrument which is used to measure the strength and directions of the magnetic field of the earth. Since the magnetometer can also be used as metal detectors which detect only magnetic metals.

\section{NEED OF ITS IN INDIA}

The economy of India as compared to world is rapidly increasing thereby increasing the use of automobiles on Indian urban roads. In 2011-12 alone, 20.4 million motorized vehicles were sold in India [18]. While a small scale ITS projects had been introduced in various cities in India including Delhi, Pune, Bangalore, Chennai and Indore for toll collection, parking information, web based traveler information etc. [19]. Apart from these application which had been deployed, there are more ITS concepts which will be useful for Indian scenario like emergency management, congestion management, advanced traffic management systems, advanced traveler information system, commercial vehicle operations, advanced vehicle control systems etc. The existing applications shows an initial potential for the 
deployment of ITS in India. But there are some challenges in the deployment of ITS in India. ITS implementation cannot be carried out by reproducing what is already been done in the developed countries because of the range of cultural, lifestyle and physical differences among them. In India the diverse range of vehicular velocities (pedestrian, bicycle, LMV's, HMV's, animal drawn carts), wide variety of vehicles (including pedestrian traffic) and poor lane discipline (partially resulting from the first two factors and partially due to cultural reasons) and very high population density makes adoption of Western ITS standards and architecture difficult.

Some of the actions needed to be taken to meet the challenges of ITS in India which include the settings up the fully functional Traffic Management Centre, developing national ITS data archive, developing models and algorithms for ITS, evolving national ITS standard for different ITS applications and do interaction between academia, industries and governmental agencies to generate more interest. These can be achieved through the improvement of technology, infrastructure and social schemes.

In India there is need to improve the roads and highway infrastructure to reduce down the traffic on congested routes. In metropolitan cities the issues been solved by building flyovers and widening the roads. But the infrastructure is restricted by the space constraints of roads of India.

\section{CONCLUSION}

Traffic in India can be characterized by the heavy congestion, poor quality of roads and disorderly traffic. ITS techniques that were developed for the developed countries, doesn't hold here. ITS in the Indian scenario fails attributable to lack of freeways, non-uniformity of vehicle speed and the lanes made by the ITS techniques in the developed regions. Installation of fixed sensor techniques involve huge installation and maintenance cost. There is need for the techniques that take the characteristics of the Indian roads into account. Use of sensors like GPS, Microphone, WI-Fi and camera in the smartphones can be used to predict the traffic conditions and arrival time of the vehicle at the destination. Installing the infrastructure on the Indian roads is restricted by the space. Since there are lot of challenges remain to be resolved for the fully deployment of ITS on Indian roads.

\section{REFERENCES}

[1] Sen, Rijurekha, Vishal Sevani, Prashima Sharma, Zahir Koradia, and Bhaskaran Raman. "Challenges In Communication Assisted Road Transportation Systems for Developing Regions." NSDR, 2009.

[2] Biagioni, James, Tomas Gerlich, Timothy Merrifield, and Jakob Eriksson. "Easytracker: automatic transit tracking, mapping, and arrival time prediction using smartphones." In Proceedings of the 9th ACM Conference on Embedded Networked Sensor Systems, pp. 68-81. ACM, 2011.

[3] Mohan, Prashanth, Venkata N. Padmanabhan, and Ramachandran Ramjee. "Nericell: rich monitoring of road and traffic conditions using mobile smartphones." In Proceedings of the 6th ACM conference on Embedded network sensor systems, pp. 323-336. ACM, 2008.

[4] Eriksson, Jakob, Lewis Girod, Bret Hull, Ryan Newton, Samuel Madden, and Hari Balakrishnan. "The pothole patrol: using a mobile sensor network for road surface monitoring." In Proceedings of the 6th international conference on Mobile systems, applications, and services, pp. 29-39. ACM, 2008.

[5] Roy, S.; Sen, R.; Kulkarni, S.; Kulkarni, P.; Raman, B.; Singh, L.K., "Wireless across road: RF based road traffic congestion detection," Communication Systems and Networks (COMSNETS), 2011 Third International Conference on, vol., no., pp.1,6, 4-8 Jan. 2011.

[6] Bhoraskar, R.; Vankadhara, N.; Raman, B.; Kulkarni, P., "Wolverine: Traffic and road condition estimation using smartphone sensors," Communication Systems and Networks (COMSNETS), pp.1,6, 2012

[7] P. Angin, B. Bhargava, and S. Helal. A Mobile Cloud Collaborative Traffic Lights Detector for Blind Navigation. First MDM international Workshop on Mobile Cloud, 2010

[8] Thiagarajan, Arvind, Lenin Ravindranath, Katrina LaCurts, Samuel Madden, Hari Balakrishnan, Sivan Toledo, and Jakob Eriksson. "VTrack: accurate, energyaware road traffic delay estimation using mobile phones." In Proceedings of the 7th ACM Conference on Embedded Networked Sensor Systems, pp. 85-98. ACM, 2009.

[9] J. Chen, M. Arumaithurai, L. Jiao, X. Fu, and K. K. Ramakrishnan. COPSS: An Efficient Content Oriented Publish/Subscribe System. ANCS, 2011.

[10] William Fenner, Michael Rabinovich, K. K. Ramakrishnan in XTreeNet: Scalable Overlay Networks for XML Content Dissemination and Querying (Synopsis). WCW, 2005.

[11] Maximilian Ott, Leslie French, Rajeev Mago, and Dhananjay Makwana in XML-based Semantic Multicast Routing: An Overlay Network Architecture for Future Information Services. IEEE Communications Society Globecom 2004.

[12] Timothy Hunter, Teodor Moldovan, Matei Zaharia, Samy Merzgui, Justin Ma, Michael J. Franklin, Pieter Abbeel, Alexandre M. Bayen in Scaling the Mobile Millennium System in the Cloud. SOCC'11, 2011.

[13] Dean Zabrieszach in "ITS activity in Australia". In ISO TC 204, WG 9 Meeting, Seattle, Washington, USA, 2013.

[14] 19th ITS World Congress, "Congress Report". In Vienna, Austria, 2012.

[15] http://www.mira.co.uk

[16] Bret Hull, Vladimir Bychkovsky, Yang Zhang, Kevin Chen, Michel Goraczko, Allen Miu, Eugene Shih, Hari Balakrishnan and Samuel Madden in CarTel: A Distributed Mobile Sensor Computing System SenSys, 2006.

[17] V. Jacobson, D. K. Smelters, J. D. Thornton, M. F. ,Plass, N. H. riggs, and R. L. Braynard. Networking Named Content. CoNEXT, 2009.

[18] http://www.knowindia.net/auto.html

[19] http://www.unescap.org/tid/projects/tiri_nag.pdf

[20] http://www.its.dot.gov/Efm/

[21] http://www.dimts.in/Traffic_Updates.aspx

[22] Jiawei Wang, Stephen Shaoyi Liao and J Zhongsheng Hua. A Hybrid Approach for Automatic Incident Detection. IEEE, 2013. 